\title{
Antihypertensive therapy improves insulin resistance and serum levels of interleukin-6 and -10 in spontaneously hypertensive rats with steatohepatitis
}

\author{
MASAYA KOZONO $^{1}$, HIROFUMI UTO ${ }^{1,2}$, RIE IBUSUKI ${ }^{1,3}$, SHIHO ARIMA $^{1}$, KOHEI ODA $^{1}$, \\ HIROKI TAGUCHI $^{1}$, FUMISATO SASAKI ${ }^{1}$, YUICHIRO NASU ${ }^{1}$, SHINICHI HASHIMOTO ${ }^{1}$, \\ HITOSHI SETOYAMA ${ }^{1}$, SHUJI KANMURA ${ }^{1}$, MASATSUGU NUMATA ${ }^{1}$, \\ HIROHITO TSUBOUCHI ${ }^{4,5}$ and AKIO IDO ${ }^{1,4}$
}

\author{
${ }^{1}$ Digestive and Lifestyle Diseases, Department of Human and Environmental Sciences, \\ Kagoshima University Graduate School of Medical and Dental Sciences, Kagoshima, Kagoshima 890-8544; \\ ${ }^{2}$ Center for Digestive and Liver Diseases, Miyazaki Medical Center Hospital, Miyazaki, Miyazaki 880-0003; \\ Departments of ${ }^{3}$ International Island and Community Medicine and ${ }^{4}$ HGF Tissue Repair and Regenerative Medicine, \\ Kagoshima University Graduate School of Medical and Dental Sciences, Kagoshima, Kagoshima 890-8544; \\ ${ }^{5}$ Kagoshima City Hospital, Kagoshima, Kagoshima 890-8760, Japan
}

Received August 18, 2015; Accepted September 1, 2016

DOI: $10.3892 / \mathrm{mmr} .2016 .5875$

\begin{abstract}
Metabolic syndrome based on insulin resistance (IR) and hypertension is a risk factor for advanced liver disease and cardiovascular disease in patients with nonalcoholic steatohepatitis (NASH). The present study investigated the effects of severe hypertension induced by a high-salt (HS) diet and antihypertensive therapy on the pathophysiological condition of spontaneously hypertensive rats (SHRs) with steatohepatitis. Steatohepatitis was induced using a choline-deficient, L-amino acid-defined diet (CDAA). Male SHRs (7-week-old) were randomly divided into five groups: Those receiving 6 weeks of standard chow with a normal salt concentration, followed by an additional 8 weeks of standard chow or CDAA with a normal salt concentration (control and CDAA groups, respectively); and those receiving 6 weeks of standard chow with HS, followed by CDAA with HS for an additional 8 weeks, with or without the antihypertensive agents, amlodipine (Aml) or hydralazine. In the CDAA and CDAA+HS groups, blood pressure was significantly correlated with serum levels of insulin, fasting blood glucose and homeostasis model assessment (HOMA)-IR. Antihypertensive therapy ameliorated the elevated glucose, insulin and HOMA-IR. Furthermore, the
\end{abstract}

Correspondence to: Dr Hirofumi Uto, Center for Digestive and Liver Diseases, Miyazaki Medical Center Hospital, 2-16 Takamatsu-cho, Miyazaki, Miyazaki 880-0003, Japan

E-mail: hirouto@m2.kufm.kagoshima-u.ac.jp

Key words: hypertension, insulin resistance, interleukin-6, interleukin-10, nonalcoholic fatty liver disease, nonalcoholic steatohepatitis, regulatory $\mathrm{T}$ cell increased levels of serum interleukin (IL)- 6 following the CDAA+HS diet were attenuated by antihypertensive therapy. The serum levels of IL-10 were increased by antihypertensive therapy, and the decrease in the proportion of splenic $\mathrm{CD} 4^{+} \mathrm{CD} 25^{+}$forkhead box $\mathrm{P}^{+} \mathrm{T}$ cells observed following the CDAA+HS diet tended to be restored by Aml. In conclusion, antihypertensive therapy improved glucose metabolism and imbalances in cytokine expression in the rat model of hypertension with steatohepatitis, suggesting that antihypertensive therapy acting through immunological factors may be beneficial for patients with metabolic syndrome-associated NASH.

\section{Introduction}

Nonalcoholic fatty liver disease (NAFLD), which includes nonalcoholic steatohepatitis (NASH), is one of the hepatic manifestations of metabolic syndrome, and is becoming a public health concern as NAFLD and NASH progress to hepatic failure and hepatocellular carcinoma (1-3). Epidemiological studies have demonstrated that $10 \%$ of patients with NASH develop cirrhosis over a 15 -year period (4), and NAFLD is a common reason for liver transplantation in the United States (5). By contrast, nonalcoholic fatty liver, or simple steatosis, rarely progresses to advanced disease. The mechanism underlying the occurrence and progression of NAFLD is currently considered to be a 'multiple hit process' involving insulin resistance (IR), oxidative stress, apoptosis and perturbations of adipokine levels $(6,7)$. Predictive factors of advanced fibrosis in patients with NAFLD also include increasing age, type 2 diabetes mellitus, and hypertension. However, the precise mechanism underlying the progression of NAFLD remains to be fully elucidated.

Metabolic syndrome is associated with IR and other metabolic risk factors, including central obesity, dyslipidemia and 
hypertension. The coexistence of these disorders is correlated with cardiovascular disease (CVD) (8-11). IR is also important in the pathogenesis of NAFLD, and patients with NAFLD have reduced insulin sensitivity, not only in muscle, but also in the liver (12). In addition, NAFLD is an independent risk factor for CVD and predicts future cardiac events (13). It was previously reported that there is an apparent association between non-obese hypertensive patients with NAFLD and increases in IR (14). The increased risk of all-cause mortality rates in patients with NAFLD appears to be associated with hypertension in addition to IR, however, the association between hypertension and IR in patients with NAFLD remains to be fully elucidated.

Our previous study (15) investigated whether high-salt (HS) diet-induced hypertension exacerbated the pathophysiology of steatohepatitis induced by a choline-deficient, L-amino acid-defined (CDAA) diet in an animal model. It was found that hypertension was a potential risk factor for liver injury and hepatic fibrosis. Using spontaneously hypertensive rats (SHRs) fed a CDAA diet, it was demonstrated that the levels of alanine aminotransferase (ALT) and alkaline phosphatase (ALP) were significantly higher, and hepatic mRNA levels of interleukin (IL)-10 were significantly lower in the HS group, compared with the normal-salt group. Immune function analysis showed that healthy human subjects on a HS diet (12 g/day) had higher levels of proinflammatory cytokines, IL-6 and IL-23, and lower levels of the anti-inflammatory cytokine, IL-10, compared with those receiving the recommended salt intake (6 g/day), suggesting that a HS diet may contribute to an excessive immune response (16). Pro-inflammatory serum cytokines, including IL-6, may be increased and anti-inflammatory cytokines, including IL-10, may be decreased in NASH due to imbalanced cytokine production in the liver and/or extrahepatic production (17-21). However, the association between IR and the production of cytokines, including IL-6 and IL-10 in NAFLD remain to be fully elucidated.

In the present study, it was hypothesized that HS-induced hypertension affects IR through imbalanced cytokine production in SHRs with CDAA-diet-induced steatohepatitis, and that IR or imbalanced cytokine production can be reversed by antihypertensive therapy. It was shown that HS-induced hypertension was positively correlated with blood glucose and levels of serum insulin, and with homeostasis model assessment (HOMA)-IR, suggesting that hypertension induced IR in this NASH model. It was also demonstrated that the expression of cytokine IL-6 was correlated with hypertension and was attenuated by antihypertensive therapy, which was accompanied by the expression of IL-10. The cytokine expression and IR induced by hypertension may be associated with the progression of NASH (22), and the experimental animal model used in the present study may be useful for investigating NASH with metabolic syndrome.

\section{Materials and methods}

Animals. All animal procedures were approved by the Institutional Animal Care and Use Committee of Kagoshima University (Kagoshima, Japan). Male, 6-week-old SHRs were obtained from Charles River Laboratories (Yokohama, Japan). The rats were allowed to acclimatize to the laboratory conditions for 1 week at a constant temperature of $24^{\circ} \mathrm{C}$ with a 12-h light-dark cycle and 40-70\% humidity, and were fed standard chow (control diet) containing $0.27 \% \mathrm{NaCl}$ (CE-2; Kyudo, Kumamoto, Japan) and water ad libitum. Following the acclimatization period, the rats were divided into groups $(n=10$ per group) and fed five different diets ad libitum, as follows: 6 weeks standard chow with normal salt concentration $(0.27 \%$ $\mathrm{NaCl}$ ), followed by a standard chow or CDAA diet containing normal salt concentration for an additional 8 weeks (control and CDAA groups, respectively), and 6 weeks standard chow with a $\mathrm{HS}$ concentration $(8.0 \% \mathrm{NaCl})$, followed by a CDAA diet containing HS for an additional 8 weeks, with or without the antihypertensive agents, amlodipine (Aml; Wako, Osaka, Japan; $10 \mathrm{mg}$ /day in food) or hydralazine (Hyd; Sigma-Aldrich; Thermo Fisher Scientific, Inc., Waltham, MA, USA; 20 mg/day in food). These were termed the CDAA+HS, CDAA+HS+Aml and CDAA+HS+Hyd groups, respectively. Thus, all experiments were performed for 14 weeks and then all rats were sacrificed by exsanguination under pentobarbital anesthesia (12-18 mg/body; intraperitoneal administration; Kyoritsu Seiyaku Corporation, Tokyo, Japan). The diets were obtained from Dyets, Inc. (Bethlehem, PA, USA) and $30 \mathrm{~g}$ per day was administered to ensure equal total food intake.

Measurement of systolic blood pressure (SBP) and serum markers. SBP was determined using the tail-cuff method once every 2 weeks between 7 and 21 weeks (MK-1030; Muromachi, Tokyo, Japan).

Blood was collected by vena cava puncture following a 12-h fast and then centrifuged at $1,800 \times \mathrm{g}$ for $5 \mathrm{~min}$ at $4^{\circ} \mathrm{C}$. The resulting serum was stored at $-80^{\circ} \mathrm{C}$. Serum levels of ALT, ALP, total cholesterol and triglyceride were determined commercially at SRL, Inc. (Tokyo, Japan). Fasting blood glucose (FBG) and serum immunoreactive insulin (IRI) levels were determined using Spotchem II-Glucose (Arkray, Kyoto, Japan) and ELISA (Morinaga Institute of Biological Science, Kanagawa, Japan), respectively. HOMA-IR was calculated using the following formula: HOMA-IR $=\mathrm{FBG}$ $(\mathrm{mg} / \mathrm{dl}) \mathrm{x}$ fasting IRI $(\mu \mathrm{U} / \mathrm{ml}) / 405$. Serum levels of IL-6 and IL-10 were determined using a rat IL-6 or IL-10 Quantikine ELISA kit (R\&D Systems, Inc., Minneapolis, MN, USA).

Histological and immunohistochemical analyses. The resected livers and spleens were weighed, and thin slices were immersed in $10 \%$ neutralized formalin and embedded in paraffin to produce $4-\mu \mathrm{m}$-thick sections for staining with hematoxylin and eosin. Immunohistochemical analyses using anti-CD68 antibody (ED-1; cat. no. ab31630; Abcam, Cambridge, MA, USA) were performed using the paraffin-embedded sections. The samples were blocked with protein block (Dako Japan Co., Ltd., Tokyo, Japan) for $10 \mathrm{~min}$, followed by incubation with the primary antibody at a 1:400 dilution overnight at $4^{\circ} \mathrm{C}$. The samples were incubated with goat anti-mouse/rabbit IgG (Histofine Simple Stain Rat MAX PO MULTI; cat. no. 414191; Nichirei Biosciences, Tokyo, Japan) to detect bound antibodies. After the signal was visualized by the 3,3-diaminobenzidine, the $\mathrm{CD}^{+} 8^{+}$cells were counted (magnification, $\mathrm{x} 400$ ) in five randomly selected fields of the periportal and perivenular areas of the liver lobules using a fluorescence microscope (BZ-9000; Keyence Corporation, Osaka, Japan). 
Table I. Body, liver and spleen weights, and SBP at week 14 in each group.

\begin{tabular}{lccccc}
\hline Group & $\begin{array}{c}\text { Body } \\
\text { weight }(\mathrm{g})\end{array}$ & $\begin{array}{c}\text { Liver } \\
\text { weight }(\mathrm{g})\end{array}$ & $\begin{array}{c}\text { Liver/body } \\
\text { weight }(\%)\end{array}$ & $\begin{array}{c}\text { Spleen } \\
\text { weight }(\mathrm{g})\end{array}$ & $\begin{array}{c}\text { SBP } \\
(\mathrm{mmHg})\end{array}$ \\
\hline Control & $357.00 \pm 19.93$ & $10.23 \pm 0.60$ & $2.87 \pm 0.09$ & $0.66 \pm 0.07$ & $201.80 \pm 8.95$ \\
CDAA & $362.40 \pm 17.89$ & $9.87 \pm 0.59$ & $2.72 \pm 0.11$ & $0.64 \pm 0.03$ & $198.12 \pm 13.03$ \\
CDAA+HS & $325.80 \pm 17.83^{\mathrm{b}, \mathrm{e}}$ & $10.40 \pm 1.01$ & $3.18 \pm 0.21^{\mathrm{c}, \mathrm{f}}$ & $0.64 \pm 0.08$ & $211.80 \pm 9.56$ \\
CDAA+HS+Aml & $334.30 \pm 20.33^{\mathrm{d}}$ & $9.63 \pm 0.58$ & $2.89 \pm 0.17^{\mathrm{g}}$ & $0.62 \pm 0.05$ & $166.16 \pm 12.48^{\mathrm{c}, \mathrm{fh}}$ \\
CDAA+HS+Hyd & $329.90 \pm 15.42^{\mathrm{a}, \mathrm{e}}$ & $10.00 \pm 0.64$ & $3.03 \pm 0.16^{\mathrm{e}}$ & $0.59 \pm 0.07$ & $170.42 \pm 16.14^{\mathrm{c}, \mathrm{h}, \mathrm{h}}$ \\
\hline
\end{tabular}

Values are presented as the mean \pm standard deviation. ${ }^{\mathrm{a}} \mathrm{P}<0.05,{ }^{\mathrm{b}} \mathrm{P}<0.01$ and ${ }^{\mathrm{c}} \mathrm{P}<0.001$, vs. Control group; ${ }^{\mathrm{d}} \mathrm{P}<0.05$, ${ }^{\mathrm{e}} \mathrm{P}<0.01$ and ${ }^{\mathrm{f}} \mathrm{P}<0.001$, vs. CDAA group; ${ }^{\mathrm{g}} \mathrm{P}<0.01$ and ${ }^{\mathrm{h}} \mathrm{P}<0.001$, vs. CDAA+HS group. CDAA, choline-deficient, L-amino acid-defined diet; HS, high-salt diet; Aml, amlodopine; Hyd, hydralazine; SBP, systolic blood pressure.

Measurement of the levels of hepatic monocyte chemoattractant protein-1 (MCP-1). The protein concentration was standardized at $8 \mathrm{mg} / \mathrm{ml}$ following extraction of total protein from the liver tissues according to a previous report (23). Rat MCP-1 in the homogenate was measured using an ELISA kit (R\&D Systems, Inc.) according to the manufacturer's protocol.

Cell isolation and flow cytometry. Single-cell suspensions of splenic cells were prepared using cell strainers and incubated for $5 \mathrm{~min}$ in $1 \mathrm{X}$ RBC lysis buffer (eBioscience, Inc., San Diego, CA, USA). The cells were washed twice using flow cytometry staining buffer (eBioscience, Inc.) and resuspended in flow cytometry staining buffer (eBioscience, Inc.). The splenic cells were analyzed by three-color intracellular flow cytometry using fluorescein isothiocyanate-CD4 (1:100; cat. no. 11-0040; $4^{\circ} \mathrm{C}, 20 \mathrm{~min}$ incubation), phycoerythrin-CD25 (1:100; cat. no. 12-0390; $4^{\circ} \mathrm{C}, 20 \mathrm{~min}$ incubation) and allophycocyanin-forkhead box P3 (Foxp3; 1:100; cat. no. 77-5775; room temperature, $30 \mathrm{~min}$ incubation) antibodies (eBioscience, Inc.). The expression of antigens were analyzed on a CyAnTM ADP flow cytometer (Beckman Coulter, Inc., Brea, CA, USA).

Statistical analysis. Data are presented as mean \pm standard deviation, or the 10th, 25th, 50th (median), 75th and 90th percentiles. Statistical comparisons among groups were performed using one-way analysis of variance followed by Tukey's post-hoc test. Correlations between continuous variables were calculated using Pearson's correlation. The $\chi^{2}$ test was used to compare the categorical variables among groups. All analyses were performed using SPSS v.18 (SPSS, Inc., Chicago, IL, USA). $\mathrm{P}<0.05$ was considered to indicate a statistically significant difference.

\section{Results}

Antihypertensive therapy in SHRs fed a HS CDAA diet. The SBP of the animals in each group were assessed between 0 and 14 weeks. The SBPs in the control and CDAA groups gradually increased in a similar manner (Fig. 1). In addition, as our previous study showed (15), administration of a HS diet induced severe hypertension. By contrast, the antihypertensive agents, Aml and Hyd, significantly decreased SBP (Fig. 1).

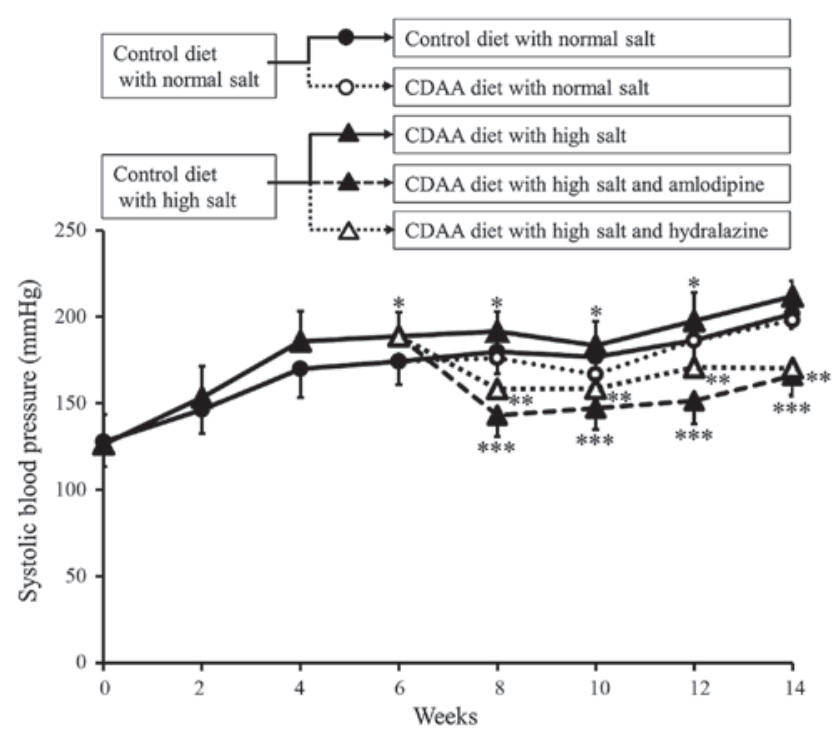

Figure 1. Changes in SBP among the five experimental groups. SHRs were fed standard CE-2 chow (Control) or a CDAA diet with or without HS Groups of SHRs with HS were also concurrently treated with an antihypertensive agent (amlodipine or hydralazine). SBP was measured using the tail-cuff method. SBP gradually increased in the rats fed HS chow, and was significantly decreased by antihypertensive therapy. Data are presented as mean \pm standard deviation. ${ }^{*} \mathrm{P}<0.05, \mathrm{CDAA}$ diet with normal salt vs. CDAA diet with high salt; ${ }^{* *} \mathrm{P}<0.05$, CDAA diet with high salt vs. CDAA diet with high salt and hydralazine; ${ }^{* * *} \mathrm{P}<0.05$, CDAA diet with high salt vs. CDAA diet with high salt and amlodipine. SBP, systolic blood pressure; SHR, spontaneously hypertensive rat; CDAA, choline-deficient, L-amino acid-defined diet; HS, high-salt diet.

At week 14, the liver weight to body weight ratio in the CDAA+HS group was significantly higher, compared with the ratios in the control and CDAA groups, and was decreased by antihypertensive therapy (Table I).

Association between SBP and blood chemistry analysis. Using a similar model as that used in the present study, our previous study (15) reported that serum levels of ALT and ALP were significantly higher in the HS group, compared with the normal-salt group. In the present study, the correlation between SBP and serum levels of ALT and ALP were investigated further. In the CDAA and CDAA+HS groups, SBP was significantly correlated with ALT and ALP (Fig. 2A and B), as expected, and with total cholesterol (TC) and 
A

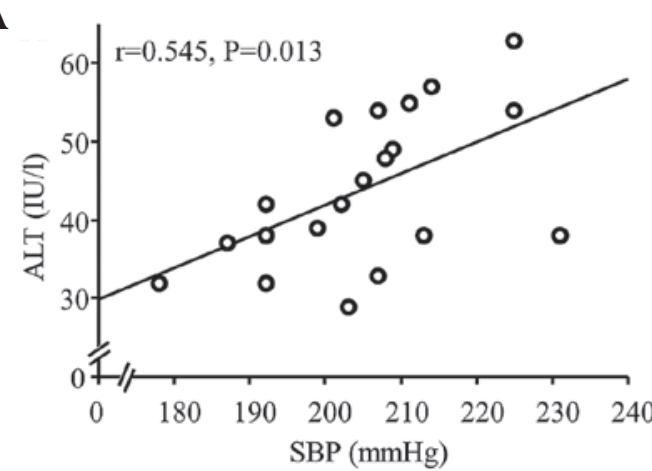

C

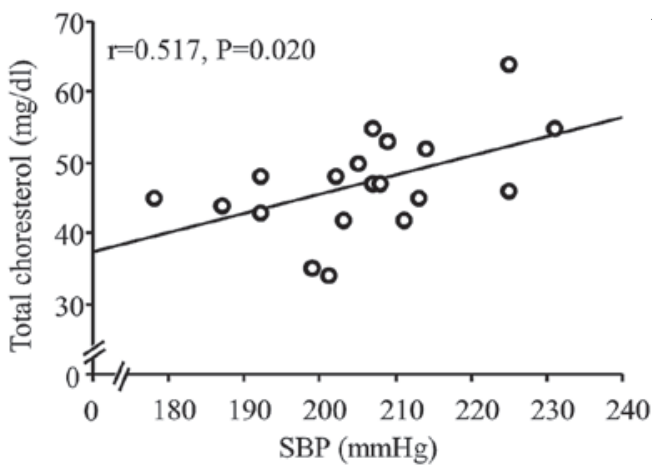

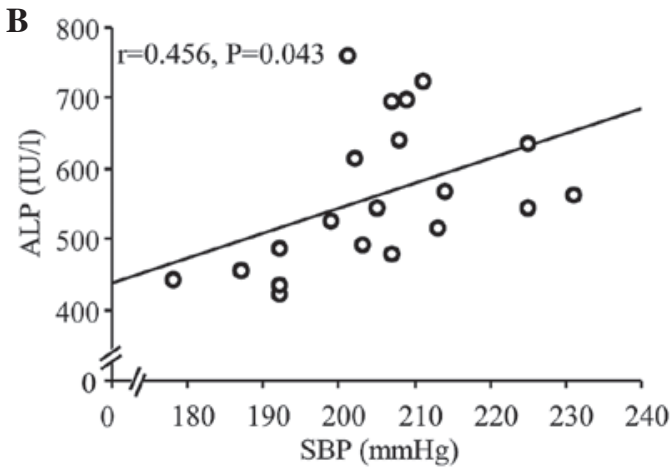

D

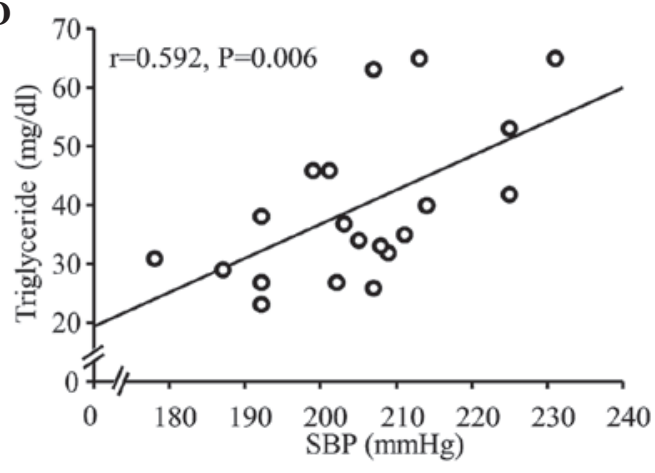

Figure 2. Correlation between SBP and blood chemistry analysis among CDAA and CDAA+HS groups. Serum levels of (A) ALT, (B) ALP, (C) total cholesterol and (D) triglyceride were significantly correlated with SBP. SBP, systolic blood pressure; CDAA, choline-deficient, L-amino acid-defined diet; HS, high-salt diet; ALT, alanine aminotransferase; ALP, alkaline phosphatase.

A

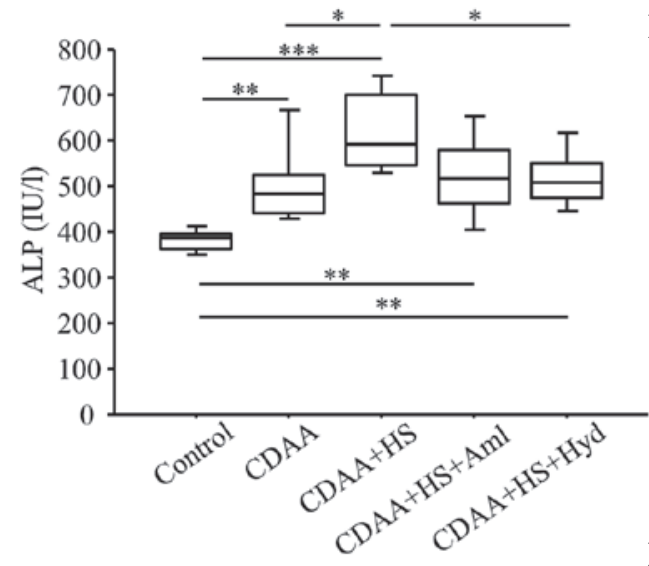

C

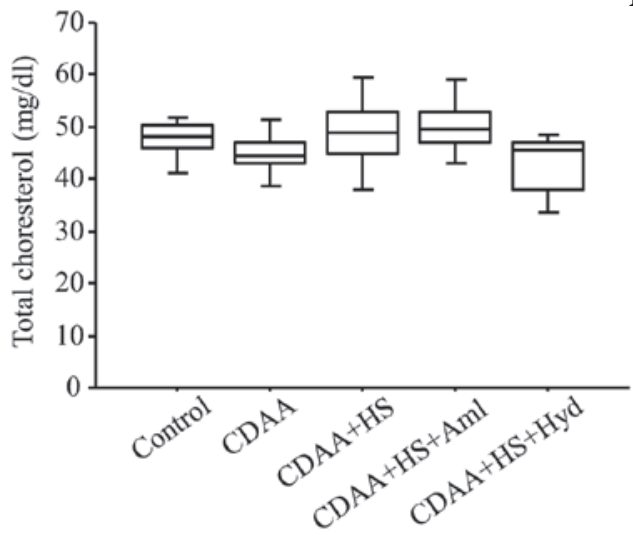

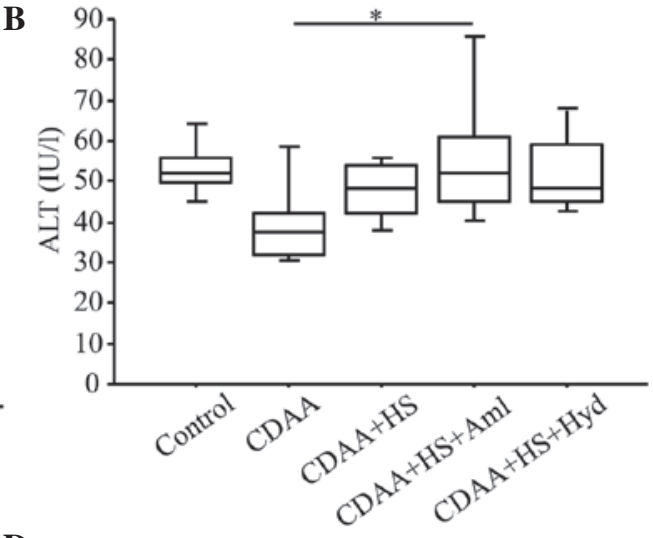

D

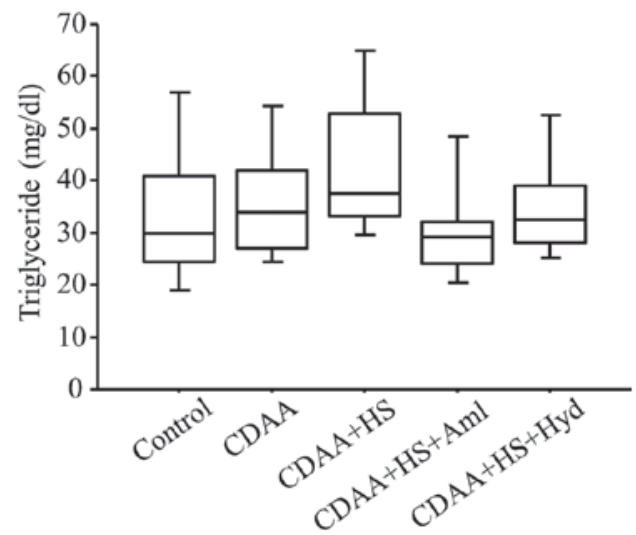

Figure 3. Serum levels of ALT, ALP, total cholesterol and triglyceride at week 14 (with or without CDAA, HS or anti-hypertensive agents). (A) Serum levels of ALP were significantly higher in the CDAA+HS group, compared with the Control and CDAA groups. Elevation of serum levels of ALP were significantly attenuated by antihypertensive therapy. These changes in ALP were not observed with serum levels of (B) ALT, (C) total cholesterol or (D) triglyceride. Box plots show the 25th, 50th (median), and 75th percentiles, with whiskers representing the 10th and 90th percentiles ( $\mathrm{n}=10$ in each group). ${ }^{*} \mathrm{P}<0.05$, ${ }^{* *} \mathrm{P}<0.01$ and ${ }^{* * * *} \mathrm{P}<0.001$. ALT, alanine aminotransferase; ALP, alkaline phosphatase; CDAA, choline-deficient, L-amino acid-defined diet; HS, high-salt diet. Aml, amlodipine; Hyd, hydralazine. 
A

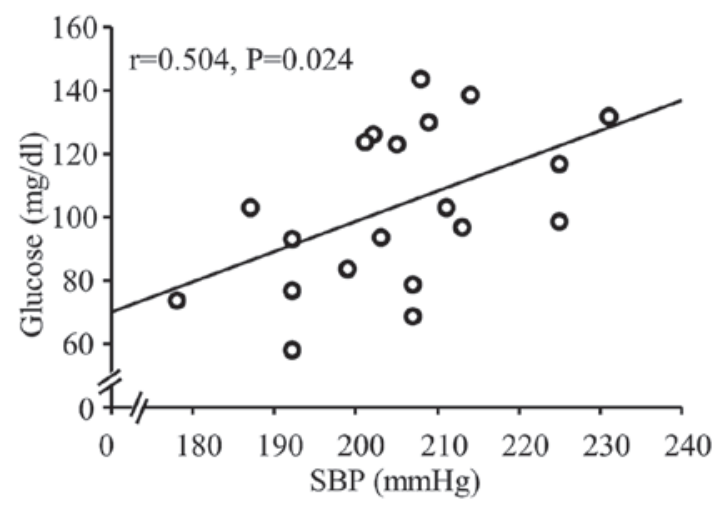

B

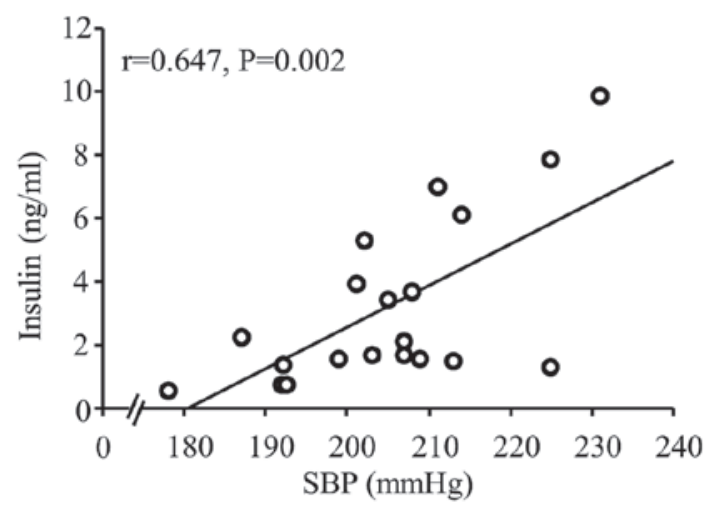

C

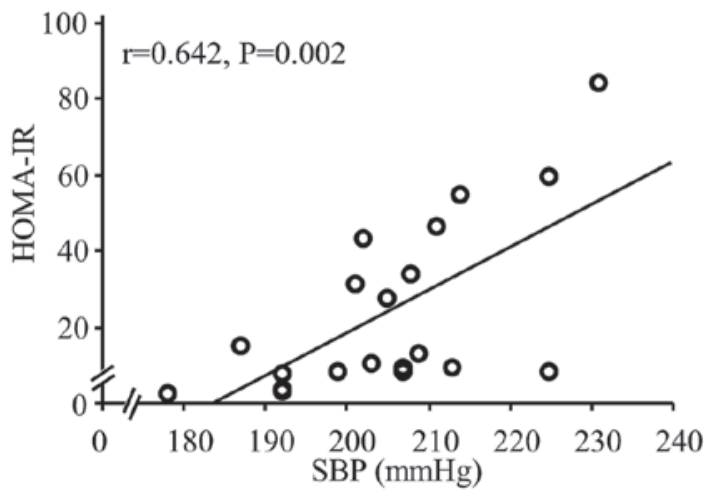

Figure 4. Correlation between SBP and glucose, insulin and HOMA-IR in the CDAA and CDAA+HS groups. (A) Fasting blood glucose, (B) serum insulin and (C) HOMA-IR were significantly correlated with SBP. SBP, systolic blood pressure; HOMA-IR, homeostasis model assessment-insulin resistance; CDAA, choline-deficient, L-amino acid-defined diet; HS, high-salt diet.

triglyceride (TG; Fig. 2C and D). Antihypertensive therapy lowered serum levels of ALP (Fig. 3A), but did not lower serum levels of ALT (Fig.3B). The serum levels of TC and TG were also reduced, although not significantly (Fig. 3C and D).

Effects of antihypertensive therapy on blood chemistry analysis. Our previous study (15) reported that FBG levels were significantly higher in the HS group, compared with the normal-salt group. The present study examined the effect of antihypertensive therapy on IR in SHRs fed a CDAA diet. Serum levels of glucose and insulin, and HOMA-IR were significantly correlated with SBP among the rats fed either a normal-salt or HS CDAA diet (Fig. 4), however, the CDAA
A

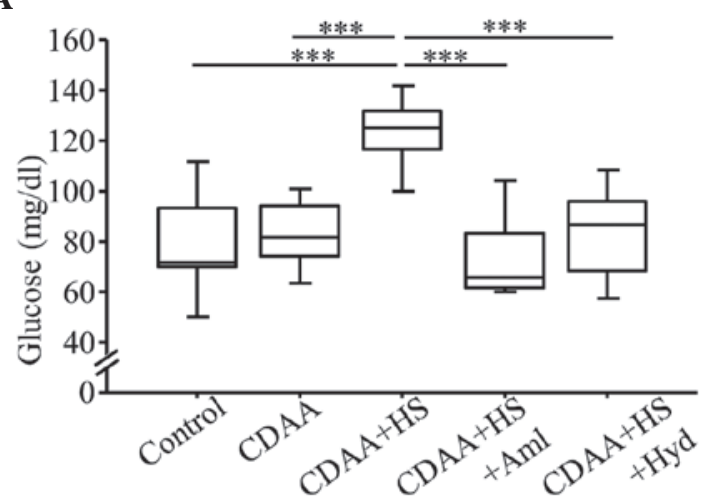

B

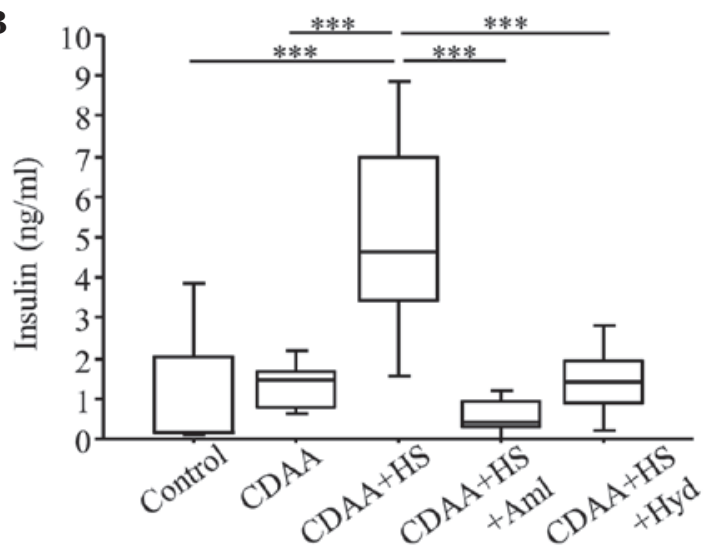

C

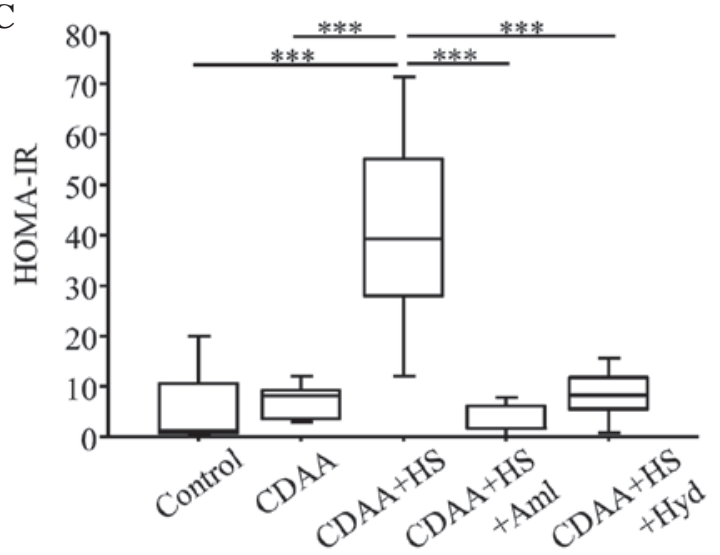

Figure 5. Fasting blood glucose, serum insulin and HOMA-IR at week 14 (with or without CDAA, HS or anti-hypertensive agents). (A) Fasting glucose and (B) serum insulin in the CDAA+HS group were higher, compared with those in the Control and CDAA groups. Increased glucose and insulin levels in the CDAA+HS group were attenuated by antihypertensive therapy with Aml or Hyd. (C) Similarly, the CDAA+HS diet induced IR, as assessed by HOMA-IR, and this IR was ameliorated by antihypertensive therapy. Box plots show the 25th, 50th (median), and 75th percentiles, with whiskers representing the 10th and 90th percentiles ( $\mathrm{n}=10$ in each group). ${ }^{* * * *} \mathrm{P}<0.001$. HOMA-IR, homeostasis model assessment-insulin resistance; CDAA, choline-deficient, L-amino acid-defined diet; HS, high-salt diet; Aml, amlodipine; Hyd, hydralazine.

diet did not affect these levels in the SHRs (control, vs. CDAA; Fig. 5). In addition, HS increased HOMA-IR in the SHRs fed a CDAA diet, indicating IR (Fig. 5C). Of note, treatment with the antihypertensive agents, Aml and Hyd, caused a significant decrease in serum levels of glucose and insulin (Fig. 5A and B), and significantly ameliorated HOMA-IR (Fig. 5C). 
A

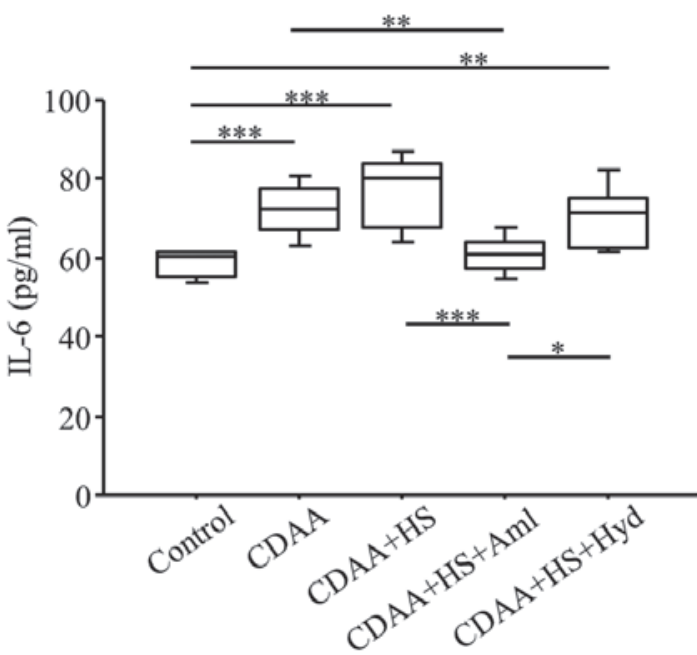

B

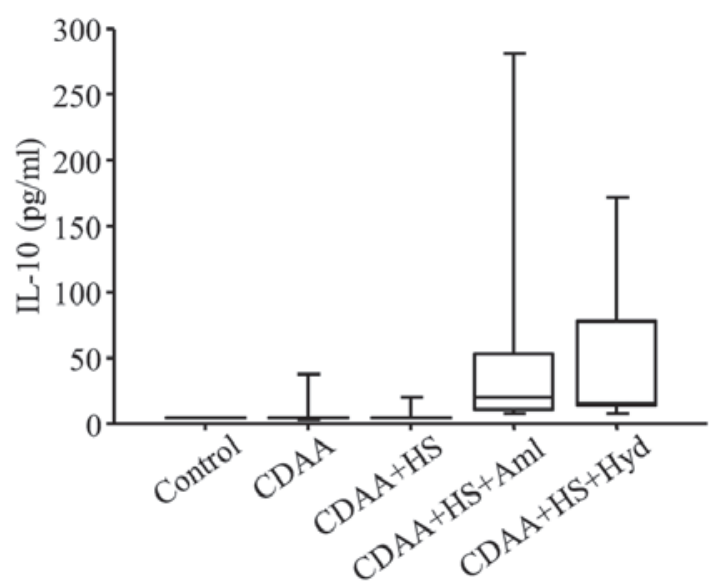

Figure 6. Serum levels of IL-6 and IL-10. Serum levels of (A) IL-6 in the CDAA and CDAA+HS groups were higher, compared with those in the Control group. Increased serum levels of IL-6 in the CDAA or CDAA+HS groups were attenuated by antihypertensive therapy with Aml or Hyd. (B) CDAA or CDAA+HS diets did not affect the serum levels of IL-10, but antihypertensive therapy increased serum levels of IL-10. Box plots show the 25th, 50th (median), and 75th percentiles, with whiskers representing the 10th and 90th percentiles ( $\mathrm{n}=10$ in each group). $\mathrm{P}<0.05,{ }^{* *} \mathrm{P}<0.01$ and ${ }^{* * * *} \mathrm{P}<0.001$. IL, interleukin; CDAA, choline-deficient, L-amino acid-defined diet; HS, high-salt diet; Aml, amlodipine; Hyd, hydralazine.

Antihypertensive therapy reduces levels of IL-6 and induces levels of IL-10 in SHRs fed a HS CDAA diet. The serum levels of IL-6 were increased in the SHRs fed a CDAA diet, and HS further increased these levels (Fig. 6A). By contrast, antihypertensive therapy with Aml significantly decreased the elevated levels of serum IL-6 induced by the CDAA and HS diets, and Hyd had a similar, although less pronounced, effect (Fig. 6A). Neither the normal nor the HS CDAA diet affected serum levels of IL-10 in the SHRs (Fig. 6B), however, antihypertensive therapy increased the levels of IL-10 in the HS groups (Fig. 6B). The number of SHRs with a level of IL-10 $>10 \mathrm{pg} / \mathrm{ml}$ was significantly higher when either Aml $(\mathrm{n}=8$; $80 \%)$ or Hyd $(\mathrm{n}=8 ; 80 \%)$ was administered with a HS CDAA diet, compared with the rats fed the HS CDAA diet only $(n=2$; $20 \% ; \mathrm{P}<0.01)$.

Increased $C D 68^{+}$cells and protein levels of $M C P-1$ in the liver are decreased by Aml in SHRs fed a HS CDAA diet. There was a significant increase in the number of $\mathrm{CD} 68^{+}$cells in the liver of the SHRs fed a HS CDAA diet, compared with the control groups (Fig. 7A). By contrast, antihypertensive therapy with $\mathrm{Aml}$ tended to decrease the elevated number of $\mathrm{CD}^{2} 8^{+}$ cells induced by the CDAA and HS diets. In addition, hepatic protein levels of MCP-1 in the liver tissue were increased in the SHRs fed a CDAA diet, and HS further increased these levels (Fig. 7B). Antihypertensive therapy with Aml significantly decreased the elevated levels of MCP-1 caused by the CDAA and HS diets.

$T$ cell profiles in the spleen of SHRs fed a CDAA diet. The frequency of $\mathrm{CD}^{+} \mathrm{T}$ cells in the spleen was similar regardless of diet (Fig. 8A). By contrast, the frequencies of $\mathrm{CD} 4^{+} \mathrm{CD} 25^{+}$ $\mathrm{T}$ cells and $\mathrm{CD}^{+} \mathrm{CD} 25^{+} \mathrm{Foxp}^{+} \mathrm{T}$ cells in the SHRs fed a normal or HS CDAA diet were significantly lower, compared with those in the control group (Fig. 8B and C). These decreases were ameliorated by Aml (Fig. 8B and C), however the differences were not significant.

\section{Discussion}

In the present study, a HS diet induced severe hypertension in SHRs, although SHRs are known to exhibit hypertension as they age (24). Furthermore, the combination of the HS-induced hypertension and CDAA-diet-induced steatohepatitis was associated with increased serum levels of glucose and insulin, and IR. These responses were accompanied by high levels of serum IL-6 and hepatic MCP-1 protein, and low frequencies of $\mathrm{CD} 4{ }^{+} \mathrm{CD} 25^{+}$and $\mathrm{CD} 4{ }^{+} \mathrm{CD} 25^{+} \mathrm{Foxp} 3^{+} \mathrm{T}$ cells in the spleen. Furthermore, antihypertensive therapy reduced the levels of proinflammatory IL- 6 and hepatic MCP-1 protein, and increased the level of anti-inflammatory IL-10. It also restored the frequencies of $\mathrm{CD} 4{ }^{+} \mathrm{CD} 25^{+}$and $\mathrm{CD} 4^{+} \mathrm{CD} 25^{+} \mathrm{Foxp} 3^{+} \mathrm{T}$ cells, although the changes in IL-10 and the indicated $\mathrm{T}$ cell frequencies were not significant. These results indicated that hypertension induced by a HS diet may cause immune-response-mediated IR in patients with NASH, suggesting one of the molecular mechanisms underlying the progression of NASH in patients with metabolic syndrome.

Pro-inflammatory cytokines, including IL-6, may be crucial in the pathogenesis and development of hypertension (25). However, it was previously reported that the deletion of IL-6 did not affect blood pressure in hypertension induced by a combination of angiotensin II and a HS diet (26). In the present study involving SHRs, a CDAA diet affected the serum levels of IL-6, but did not affect blood pressure. The effect of IL-6 on hypertension may vary among animal models and physiological conditions, and this effect may not be present in the experimental model. By contrast, the present study found that the overexpression of IL- 6 induced by a HS CDAA diet was reduced by antihypertensive therapy. Serum levels of IL-10 were increased by antihypertensive therapy, but were not affected by a HS diet. Therefore, pathways other than those involving the IL-6 signaling may have been active in HS-induced hypertension in the present study. The reduction of IL- 6 may be associated with the overexpression of IL-10 caused by antihypertensive therapy, 
$\mathbf{A}$

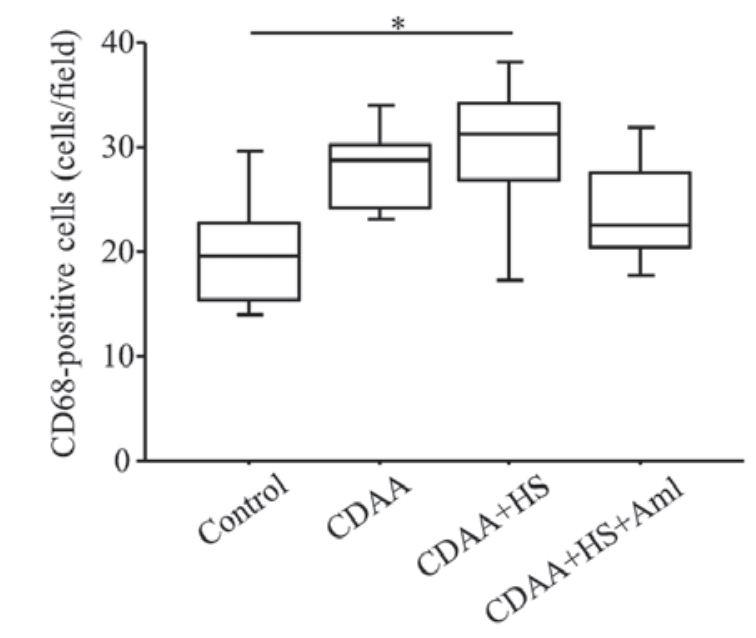

B

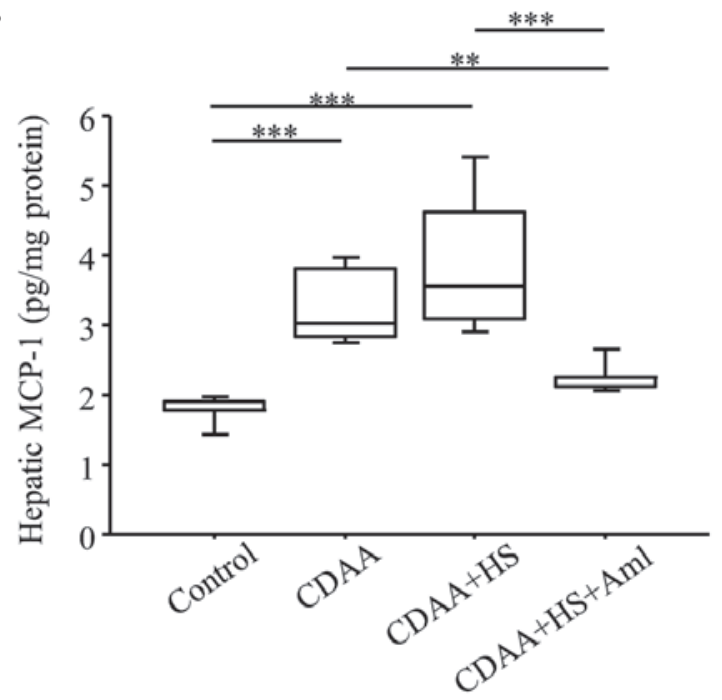

Figure 7. Quantitation of $\mathrm{CD} 68^{+}$cells and protein expression levels of MCP-1 in the liver. (A) Numbers of $\mathrm{CD} 68^{+}$cells increased significantly in SHRs fed a HS CDAA diet, compared with control groups, and increased numbers of $\mathrm{CD} 68^{+}$cells were decreased by Aml. (B) HS CDAA diet increased hepatic expression levels of MCP-1 in SHRs, and Aml significantly attenuated this effect. Box plots show the 25th, 50th (median), and 75th percentiles, with whiskers representing the 10th and 90th percentiles ( $\mathrm{n}=10$ in each group). ${ }^{*} \mathrm{P}<0.05,{ }^{* * *} \mathrm{P}<0.01$ and ${ }^{* * *} \mathrm{P}<0.001$. MCP-1, monocyte chemotactic protein-1; HS, high-salt diet; CDAA, choline-deficient, L-amino acid-defined diet; Aml, amlodipine.

and these cytokine expression patterns may affect systemic pathogenesis, including IR, in NASH.

Systemic inflammation is a characteristic feature of metabolic syndrome and CVD. A common serum or plasma marker of systemic inflammation is C-reactive protein (CRP) (27). It has been reported that high-sensitivity CRP is an independent predictor of the risk of cardiovascular events (28). Furthermore, IL-6 has been reported to be an independent predictor of future cardiovascular events in high-risk Japanese patients (29). The most marked correlations between serum levels of CRP and IL-6 are observed in men with angiographically-documented coronary heart disease (30). Serum levels of IL-6 and high-sensitivity CRP are also shown to be higher in patients with NAFLD, compared with healthy
A

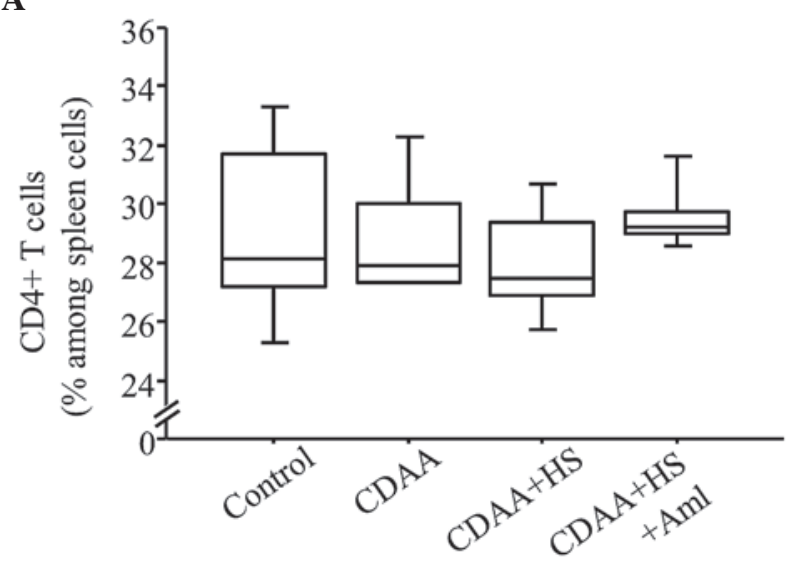

B
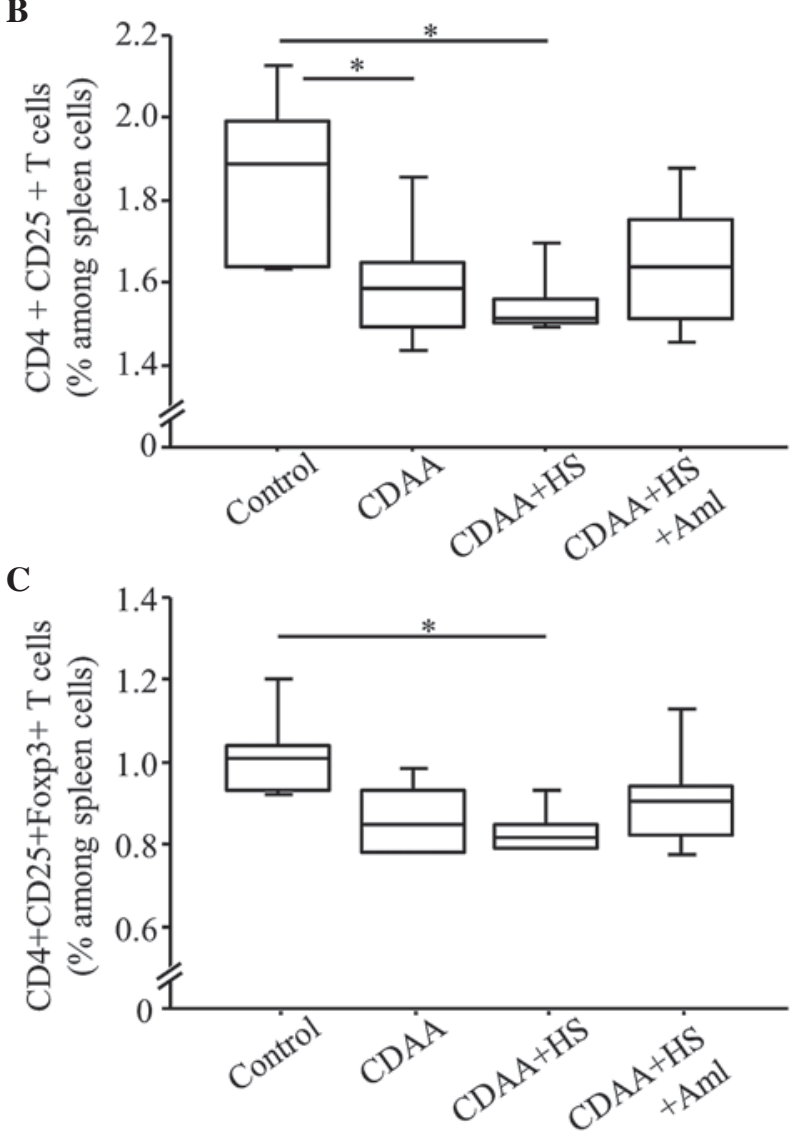

Figure $8 . \mathrm{T}$ cell profiles in the spleens of spontaneously hypertensive rats with or without CDAA and/or HS diet. The percentage of (A) $\mathrm{CD} 4^{+} \mathrm{T}$ cells, (B) $\mathrm{CD} 4{ }^{+} \mathrm{CD} 25^{+} \mathrm{T}$ cells and (C) $\mathrm{CD} 4^{+} \mathrm{CD} 25^{+} \mathrm{Foxp} 3^{+} \mathrm{T}$ cells. Phenotypes of the $\mathrm{T}$ cells from individual rats groups ( $\mathrm{n}=6$ rats per group) were determined using flow cytometry. Box plots show the 25th, 50th (median), and 75th percentiles, with whiskers representing the 10 th and 90 th percentiles. ${ }^{*} \mathrm{P}<0.05$. CDAA, choline-deficient, L-amino acid-defined diet; HS, high-salt diet; Aml, amlodipine; Foxp3, forkhead box P3.

controls $(17,31)$. Therefore, the present study hypothesized that NAFLD-associated IL-6 contributes to hypertensive heart disease, which can result in extrahepatic disease-associated mortality. Longitudinal investigations are required to investigate whether the inflammatory cytokines associated with hypertension affect mortality rates in patients with NAFLD.

It was previously reported that the short-term infusion of IL-6 does not induce IR or impair insulin signaling in rats (32). By contrast, IL-6 has been reported to induce IR in 3T3-L1 
adipocytes and be overexpressed in human fat cells from subjects with IR (33). Chronic IL-6 treatment increases the secretion of IL-6 and induces IR in adipocytes (34). In addition, the depletion of IL-6 improves insulin responsiveness in insulin tolerance tests in mice with diet-induced obesity (35). Long-term exposure to high levels of IL-6 may be required prior to IR being affected. Furthermore, Klover et al (36) suggested that the major targets for cross-talk between IL-6 and insulin may be adipose tissue and the liver, rather than skeletal muscle. In the present study, insulin signaling, assessed by the phosphorylation of insulin receptor substrate- 1 in liver tissue, was inhibited in the HS CDAA group, compared with the controls. This inhibition was attenuated by antihypertensive therapy, which was accompanied by lower serum levels of IL-6 (data not shown). Thus, although the entire molecular mechanism remains to be fully elucidated, IL- 6 and IR in the liver were linked in the present study, and may affect pathological hepatic conditions, including hepatic fibrosis (22).

IL-10 is known to have anti-inflammatory effects in various organs and tissues under pathological conditions, however, studies investigating the effect of endogenous IL-10 in the pathogenesis of NAFLD are limited (37). Kim et al (38) found that treatment with IL-10 prevents IL-6-induced defects in hepatic insulin action and signaling activity. Hong et al (39) showed that transgenic mice with muscle-specific overexpression of IL-10 are protected from diet-induced IR in skeletal muscle, and this is associated with reduced levels of cytokines, including IL-6, in the skeletal muscle. In another study, endogenous IL-10 inhibition was found to impair insulin signaling and promote the increased expression of inflammatory cytokines, including IL-6 in an animal model of liver disease induced by a high-fat diet (19). The above findings are consistent with the observations in the present study, that the induction of IL-10 and suppression of IL- 6 by antihypertensive therapy were at least partly associated with improvement of IR.

It was previously reported that mice lacking $\mathrm{T}$ and $\mathrm{B}$ cells (Rag1\%mice) do not develop hypertension, indicating that $\mathrm{T}$ cells are important in the genesis of hypertension (40). Matrougui et al (41) showed that hypertension is associated with increased numbers of apoptotic regulatory T cells (Tregs) in the spleen and a reduction in plasma IL-10 content, and that the transfer of Tregs to hypertensive animals reduces blood pressure and inhibits the reduction in serum levels of IL-10. Furthermore, Eller et al (42) indicated that Tregs are key regulatory cells in the pathogenesis of IR, and that intravenous transfer of Tregs improves IR in vivo. In the present study, the frequencies of $\mathrm{CD} 4^{+} \mathrm{CD} 25^{+}$and $\mathrm{CD} 4^{+} \mathrm{CD} 25^{+} \mathrm{Foxp} 3^{+} \mathrm{T}$ cells in the spleen were significantly reduced by a HS CDAA diet in the SHRs, and these reductions were relatively attenuated by antihypertensive therapy accompanied by high serum levels of IL-10. Thus, Tregs may be involved in the pathogenesis of IR in this model of HS-induced hypertension with steatohepatitis.

MCP-1, which is referred to as chemokine (C-C motif) ligand (CCL)2, is a potent chemoattractant, which is primarily secreted by macrophages. CCL2 has also been found to be upregulated in the livers of animals with high-fat diet-induced NASH (43). Obstfeld et al (44) showed that obesity activates the hepatocyte expression of CCL2/MCP-1, leading to hepatic recruitment of $\mathrm{CCR} 2^{+}$myeloid cells, which promote hepatosteatosis. By contrast, CCL2 deletion in an experimental model of methionine-choline-deficient diet-induced steatosis did not improve liver fat accumulation or associated inflammation (45). In the present study, neither hypertension induced by a HS diet nor antihypertensive therapy affected hepatic steatosis induced by a CDAA diet (data not shown). By contrast, our previous study found that long-term hypertension induced by a HS diet exacerbated hepatic fibrosis induced by a CDAA diet (15). In the present study, the hepatic overexpression of MCP-1 was accompanied by an increased number of $\mathrm{CD}^{+} 8^{+}$cells, indicating the presence of macrophages in the liver. Marra and Tacke (46) also suggested that during the development of NASH, CCL2 and its receptor are upregulated in the liver, where they promote macrophage accumulation, inflammation and fibrosis. Therefore, the present study hypothesized that the hepatic expression of MCP-1 is associated with hepatic inflammation and fibrosis rather than hepatic steatosis.

The present study had several limitations. First, the control rats were fundamentally hypertensive, regardless of salt concentrations in the diet. This hypertension may have affected several measurements in the controls, including the levels of ALT, IL-6 and IL-10, and this may have masked the differences between the normal and HS groups. Further experiments using other hypertension models are required to confirm the data. Secondly, the observed changes in the frequencies of $\mathrm{T}$ cell subpopulations in the spleen appeared to be too small to explain the differences in IL-6 and IL-10 between the CDAA+HS and CDAA+HS+Aml groups. T cell subpopulations in other tissues, including adipose tissue and the liver require consideration. Thirdly, antihypertensive therapy did not decrease the levels of ALT, although these levels were associated with blood pressure. However, serum levels of ALT do not always reflect the severity of steatohepatitis induced by MCD diets in mice (47). The number of CD68 $8^{+}$cells in the liver and the hepatic expression of MCP-1 may be more useful markers to evaluate improvements of steatohepatitis severity in the present study. Finally, the present study did not identify a direct association between cytokines, IR and hypertension, and did not investigate long-term hepatic fibrosis. However, our previous study showed that hypertension affected hepatic fibrosis, and the present study indicated that hypertension in the context of steatohepatitis was possibly associated with IR mediated through cytokine imbalance; these abnormalities affect the progression of steatohepatitis, which is associated with hepatic fibrosis. The experimental model used in the present study is likely to be a useful model of human NASH with metabolic syndrome.

In conclusion, the present study demonstrated that rats with HS-induced hypertension developed IR, which may have been associated with an imbalance of IL- 6 and IL-10, suppression of Tregs in the spleen and hepatic levels of MCP-1. These results may indicate the importance of cytokines and Tregs in the pathogenesis of IR in NASH with hypertension, and may lead to novel therapeutic concepts for the treatment of metabolic syndrome with NASH.

\section{Acknowledgements}

The authors would like to thank Yuko Morinaga, Etsuko Horiguchi and Ayaka Hamabe for their technical assistance. 
This study was supported in part by grants from the Ministry of Education, Culture, Sports, Science and Technology of Japan (grant no. 23590981) and the Takeda Science Foundation.

\section{References}

1. Oda K, Uto H, Mawatari S and Ido A: Clinical features of hepatocellular carcinoma associated with nonalcoholic fatty liver disease: A review of human studies. Clin J Gastroenterol 8: 1-9, 2015.

2. Watanabe S, Hashimoto E, Ikejima K, Uto H, Ono M, Sumida Y, Seike M, Takei Y, Takehara T, Tokushige K, et al: Evidence-based clinical practice guidelines for nonalcoholic fatty liver disease/nonalcoholic steatohepatitis. J Gastroenterol 50: 364-377, 2015.

3. Chalasani N, Younossi Z, Lavine JE, Diehl AM, Brunt EM, Cusi K, Charlton M and Sanyal AJ: The diagnosis and management of non-alcoholic fatty liver disease: Practice guideline by the American Association for the Study of Liver Diseases, American College of Gastroenterology and the American Gastroenterological Association. Hepatology 55: 2005-2023, 2012.

4. Angulo P: Long-term mortality in nonalcoholic fatty liver disease: Is liver histology of any prognostic significance? Hepatology 51: 373-375, 2010.

5. Charlton MR, Burns JM, Pedersen RA, Watt KD, Heimbach JK and Dierkhising RA: Frequency and outcomes of liver transplantation for nonalcoholic steatohepatitis in the United States. Gastroenterology 141: 1249-1253, 2011.

6. Tilg H and Moschen AR: Evolution of inflammation in nonalcoholic fatty liver disease: The multiple parallel hits hypothesis. Hepatology 52: 1836-1846, 2010.

7. Than NN and Newsome PN: A concise review of non-alcoholic fatty liver disease. Atherosclerosis 239: 192-202, 2015.

8. Lewington S, Clarke R, Qizilbash N, Peto R and Collins R; Prospective Studies Collaboration: Age-specific relevance of usual blood pressure to vascular mortality: a meta-analysis of individual data for one million adults in 61 prospective studies. Lancet 360: 1903-1913, 2002.

9. Yusuf S, Hawken S, Ounpuu S, Dans T, Avezum A, Lanas F, McQueen M, Budaj A, Pais P, Varigos J and Lisheng L; INTERHEART Study Investigators: Effect of potentially modifiable risk factors associated with myocardial infarction in 52 countries (the INTERHEART study): Case-control study. Lancet 364: 937-952, 2004.

10. O'Donnell MJ, Xavier D, Liu L, Zhang H, Chin SL, Rao-Melacini P, Rangarajan S,Islam S, Pais P, McQueen MJ, et al; INTERSTROKE investigators: Risk factors for ischaemic and intracerebral haemorrhagic stroke in 22 countries (the INTERSTROKE study): A case-control study. Lancet 376: $112-123,2010$

11. Hamabe A, Uto H, Imamura Y, Kusano K, Mawatari S, Kumagai K, Kure T, Tamai T, Moriuchi A, Sakiyama T, et al: Impact of cigarette smoking on onset of nonalcoholic fatty liver disease over a 10-year period. J Gastroenterol 46: 769-778, 2011

12. Marchesini G, Brizi M, Morselli-Labate AM, Bianchi G, Bugianesi E, McCullough AJ, Forlani G and Melchionda N: Association of nonalcoholic fatty liver disease with insulin resistance. Am J Med 107: 450-455, 1999.

13. Brea A and Puzo J: Non-alcoholic fatty liver disease and cardiovascular risk. Int J Cardiol 167: 1109-1117, 2013.

14. Donati G, Stagni B, Piscaglia F, Venturoli N, Morselli-Labate AM, Rasciti L and Bolondi L: Increased prevalence of fatty liver in arterial hypertensive patients with normal liver enzymes: Role of insulin resistance. Gut 53: 1020-1023, 2004.

15. Arima S, Uto H, Ibusuki R, Kumamoto R, Tanoue S, Mawatari S, Oda K, Numata M, Fujita H, Oketani M, et al: Hypertension exacerbates liver injury and hepatic fibrosis induced by a choline-deficient L-amino acid-defined diet in rats. Int J Mol Med 33: 68-76, 2014.

16. Yi B, Titze J, Rykova M, Feuerecker M, Vassilieva G, Nichiporuk I, Schelling G, Morukov B and Choukèr A: Effects of dietary salt levels on monocytic cells and immune responses in healthy human subjects: A longitudinal study. Transl Res 166: 103-110, 2015.

17. Haukeland JW, Damås JK, Konopski Z, Løberg EM, Haaland T, Goverud I, Torjesen PA, Birkeland K, Bjøro K and Aukrust P: Systemic inflammation in nonalcoholic fatty liver disease is characterized by elevated levels of CCL2. J Hepatol 44: $1167-1174,2006$
18. Wieckowska A, Papouchado BG, Li Z, Lopez R, Zein NN and Feldstein AE: Increased hepatic and circulating interleukin-6 levels in human nonalcoholic steatohepatitis. Am J Gastroenterol 103: 1372-1379, 2008.

19. Cintra DE, Pauli JR, Araújo EP, Moraes JC, de Souza CT, Milanski M, Morari J, Gambero A, Saad MJ and Velloso LA: Interleukin-10 is a protective factor against diet-induced insulin resistance in liver. J Hepatol 48: 628-637, 2008.

20. Esposito K, Pontillo A, Giugliano F, Giugliano G, Marfella R, Nicoletti G and Giugliano D: Association of low interleukin-10 levels with the metabolic syndrome in obese women. J Clin Endocrinol Metab 88: 1055-1058, 2003.

21. Braunersreuther V, Viviani GL, Mach F and Montecucco F: Role of cytokines and chemokines in non-alcoholic fatty liver disease. World J Gastroenterol 18: 727-735, 2012.

22. Hui JM, Hodge A, Farrell GC, Kench JG, Kriketos A and George J: Beyond insulin resistance in NASH: TNF-alpha or adiponectin? Hepatology 40: 46-54, 2004.

23. Sánchez-Lozada LG, Mu W, Roncal C, Sautin YY, Abdelmalek M, Reungjui S, Le M, Nakagawa T, Lan HY, Yu X and Johnson RJ: Comparison of free fructose and glucose to sucrose in the ability to cause fatty liver. Eur J Nutr 49: 1-9, 2010.

24. Varagic J, Ahmad S, Voncannon JL, Moniwa N, Simington SW Jr, Brosnihan BK, Gallagher PE, Habibi J, Sowers JR and Ferrario CM: Nebivolol reduces cardiac angiotensin II, associated oxidative stress and fibrosis but not arterial pressure in salt-loaded spontaneously hypertensive rats. J Hypertens 30: 1766-1774, 2012

25. Jurewicz M, McDermott DH, Sechler JM, Tinckam K, Takakura A, Carpenter CB, Milford E and Abdi R: Human T and natural killer cells possess a functional renin-angiotensin system: Further mechanisms of angiotensin II-induced inflammation. J Am Soc Nephrol 18: 1093-1102, 2007.

26. González GE, Rhaleb NE, D'Ambrosio MA, Nakagawa P, Liu Y, Leung P, Dai X, Yang XP, Peterson EL and Carretero OA: Deletion of interleukin-6 prevents cardiac inflammation, fibrosis and dysfunction without affecting blood pressure in angiotensin II-high salt-induced hypertension. J Hypertens 33: 144-152, 2015.

27. Bassuk SS, Rifai N and Ridker PM: High-sensitivity C-reactive protein: Clinical importance. Curr Probl Cardiol 29: 439-493, 2004.

28. Ridker PM, Hennekens CH, Buring JE and Rifai N: C-reactive protein and other markers of inflammation in the prediction of cardiovascular disease in women. N Engl J Med 342: 836-843, 2000.

29. Nishida H, Horio T, Suzuki Y, Iwashima Y, Tokudome T, Yoshihara F, Nakamura S and Kawano Y: Interleukin-6 as an independent predictor of future cardiovascular events in high-risk Japanese patients: Comparison with C-reactive protein. Cytokine 53: 342-346, 2011

30. Rifai N, Joubran R, Yu H, Asmi M and Jouma M: Inflammatory markers in men with angiographically documented coronary heart disease. Clin Chem 45: 1967-1973, 1999.

31. Riquelme A, Arrese M, Soza A, Morales A, Baudrand R, Pérez-Ayuso RM, González R, Alvarez M, Hernández V, García-Zattera MJ, et al: Non-alcoholic fatty liver disease and its association with obesity, insulin resistance and increased serum levels of C-reactive protein in Hispanics. Liver Int 29: 82-88, 2009.

32. Rotter Sopasakis V, Larsson BM, Johansson A, Holmäng A and Smith U: Short-term infusion of interleukin-6 does not induce insulin resistance in vivo or impair insulin signalling in rats. Diabetologia 47: 1879-1887, 2004.

33. Rotter V, Nagaev I and Smith U: Interleukin-6 (IL-6) induces insulin resistance in 3T3-L1 adipocytes and is, like IL-8 and tumor necrosis factor-alpha, overexpressed in human fat cells from insulin-resistant subjects. J Biol Chem 278: 45777-45784, 2003.

34. Lagathu C, Bastard JP, Auclair M, Maachi M, Capeau J and Caron M: Chronic interleukin-6 (IL-6) treatment increased IL-6 secretion and induced insulin resistance in adipocyte: Prevention by rosiglitazone. Biochem Biophys Res Commun 311: 372-379, 2003.

35. Klover PJ, Clementi AH and Mooney RA: Interleukin-6 depletion selectively improves hepatic insulin action in obesity. Endocrinology 146: 3417-3427, 2005.

36. Klover PJ, Zimmers TA, Koniaris LG and Mooney RA: Chronic exposure to interleukin- 6 causes hepatic insulin resistance in mice. Diabetes 52: 2784-2789, 2003.

37. Meda C: The impact of cytokines and chemokines on non-alcoholic fatty liver disease (NAFLD). Biotech Mol Biol Nanomed 2: 15-16, 2014. 
38. Kim HJ, Higashimori T, Park SY, Choi H, Dong J, Kim YJ Noh HL, Cho YR, Cline G, Kim YB and Kim JK: Differential effects of interleukin-6 and -10 on skeletal muscle and liver insulin action in vivo. Diabetes 53: 1060-1067, 2004.

39. Hong EG, Ko HJ, Cho YR, Kim HJ, Ma Z, Yu TY, Friedline RH, Kurt-Jones E, Finberg R, Fischer MA, et al: Interleukin-10 prevents diet-induced insulin resistance by attenuating macrophage and cytokine response in skeletal muscle. Diabetes 58: 2525-2535, 2009.

40. Guzik TJ, Hoch NE, Brown KA, McCann LA, Rahman A, Dikalov S, Goronzy J, Weyand C and Harrison DG: Role of the $\mathrm{T}$ cell in the genesis of angiotensin II induced hypertension and vascular dysfunction. J Exp Med 204: 2449-2460, 2007.

41. Matrougui K, Abd Elmageed Z, Kassan M, Choi S, Nair D, Gonzalez-Villalobos RA, Chentoufi AA, Kadowitz P Belmadani S and Partyka M: Natural regulatory T cells control coronary arteriolar endothelial dysfunction in hypertensive mice. Am J Pathol 178: 434-441, 2011.

42. Eller K, Kirsch A, Wolf AM, Sopper S, Tagwerker A, Stanzl U, Wolf D, Patsch W, Rosenkranz AR and Eller P: Potential role of regulatory $\mathrm{T}$ cells in reversing obesity-linked insulin resistance and diabetic nephropathy. Diabetes 60: 2954-2962, 2011.
43. Ito M, Suzuki J, Tsujioka S, Sasaki M, Gomori A, Shirakura T, Hirose $\mathrm{H}$, Ito $\mathrm{M}$, Ishihara $\mathrm{A}$, Iwaasa $\mathrm{H}$ and Kanatani $\mathrm{A}$ : Longitudinal analysis of murine steatohepatitis model induced by chronic exposure to high-fat diet. Hepatol Res 37: 50-57, 2007.

44. Obstfeld AE, Sugaru E, Thearle M, Francisco AM, Gayet C, Ginsberg HN, Ables EV and Ferrante AW Jr: C-C chemokine receptor 2 (CCR2) regulates the hepatic recruitment of myeloid cells that promote obesity-induced hepatic steatosis. Diabetes 59: 916-925, 2010

45. Kassel KM, Guo GL, Tawfik O and Luyendyk JP: Monocyte chemoattractant protein-1 deficiency does not affect steatosis or inflammation in livers of mice fed a methionine-choline-deficient diet. Lab Invest 90: 1794-1804, 2010.

46. Marra F and Tacke F: Roles for chemokines in liver disease. Gastroenterology 147: 577-594, 2014.

47. Itagaki H, Shimizu K, Morikawa S, Ogawa K and Ezaki T: Morphological and functional characterization of non-alcoholic fatty liver disease induced by a methionine-choline-deficient diet in C57BL/6 mice. Int J Clin Exp Pathol 6: 2683-2696, 2013. 